INTERNATIONAL DESIGN CONFERENCE - DESIGN 2018

https://doi.org/10.21278/idc.2018.0477

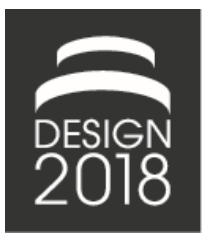

\title{
INTEGRATED SYSTEM DESIGN OF A MODULAR, AUTONOMOUS, AERIAL AND GROUND VEHICLE FLEET FOR DISASTER RELIEF MISSIONS - A CASE STUDY
}

\author{
A. C. Gärtner, D. Ferriero, A. E. Bayrak and P. Y. Papalambros
}

\begin{abstract}
We present a case study for designing a fleet of modular, unmanned aerial and ground vehicles (UAV and UGV) working cooperatively in a disaster relief mission. The study is motivated by the desire to explore the potential benefits of modularity coupled with autonomy. For example, as a result of the synergy between autonomy and modularity at the fleet level, autonomous battery replacement can extend the operation range of aerial vehicles. The study follows a simplified design process from problem definition to prototyping of the modular aerial vehicle as a first step in developing the fleet.
\end{abstract}

Keywords: modularisation, autonomous drones, disaster relief, design process, complex systems

\section{Introduction}

Natural disasters are unpredictable events that require immediate action to save human lives. Rescue resources are scarce and under time stress. Technology aids such as Unmanned Aerial Vehicles (UAVs) and Unmanned Ground Vehicles (UGVs) offer promise for disaster relief (Measure, 2015; KruijffKorbayová et al., 2016) particularly due to advances in autonomy.

In this paper, we develop a system design process for a modular, autonomously operating fleet of aerial and ground vehicles for disaster relief. This process accounts for the existence of multiple subsystems that are coupled and must be designed and operated together; it integrates ideation, configuration (topology) design, and overall 'supersystem' (top-level) design optimization; it is systematic but not automated, allowing for designer's intuition; and, it focuses on fleet-level functional and embodiment design rather than on the control algorithms needed for autonomy.

Architecture design (also referred to as configuration or topology design) for modularity has been studied mostly for single products (e.g., Gershenson et al., 2003) and for product family design (e.g., Simpson et al., 2014). Product family modularity concerns primarily manufacturing costs with no design changes during operation. Most modularity work addresses common functionality that might lead to design changes, e.g., Koh et al. $(2013 ; 2015)$ focus on reducing design changes. Bayrak et al. (2017) addressed powertrain modularity reconfigurable during system operation. There is paucity of studies in the open literature on modular systems, such as the aforementioned vehicle fleets. Autonomy research focuses largely on controls rather than design. This paper addresses the synergy between modularity and autonomy for effective design of these systems. 


\subsection{Existing uses of UAVs and UGVs}

Three types of UAVs are commonly used in disaster relief missions, namely, fixed-wing aircrafts, rotary aircrafts and blimps. Rotary aircrafts can be multi-rotor or helicopters. Fixed-wing aircrafts are fast and easy to control with high payload capacities, but cannot hover, have limited manoeuvrability, and require space to take-off and land. Rotary aircrafts have better manoeuvrability, can take-off vertically and hover, but have lower payload capacity and shorter time of operation. Blimps are slow, inflexible vehicles with low payload capacities but long operation times.

In disaster relief, UAVs and UGVs perform several tasks (Measure, 2015; Murphy et al., 2016). For example, reconnaissance and mapping tare performed by fixed wing or multi-rotor drones (Câmara, 2014); for fire outbreaks, search and rescue missions, or during chemical, biological, radiological, nuclear or explosive (CBRNE) events, UAVs are equipped with cameras and sensors to look for survivors (López et al., 2017), monitor disaster environments and measure air quality. Multi-rotor drones perform structural integrity assessment of damaged buildings with 3D models. Heavy-duty single rotor aircrafts transport goods to inaccessible areas, extinguish fires, disperse neutralizing agents in CBRNE events, and carry UGVs. The UGVs can locally transport tools and materials, map contaminants, and provide localized images and uncover victims. Blimps create Wi-Fi hotspots (Iqbal et al., 2015).

\subsection{Modular design methods}

Modular architecture design is based on creating boundaries between elements that constitute the system. Modularity decisions look to minimize interactions between modular elements (Ulrich, 1994) or to maximize functional and physical independence of modules (Gershenson et al., 2003). Design Structure Matrices (DSM) can support optimal modular architecture decisions (Huang and Kusiak, 1998). DSM clustering methods can be used for module identification (Borjesson and Hölttä-Otto, 2014).

Design of a collective supersystem consisting of individual systems working together to achieve a common goal poses several unique challenges. Design problems for individual systems are coupled and must be addressed together. Design of product families is an example to such a supersystem design problem (Fujita and Yoshida, 2004; Simpson et al., 2014). These studies do not consider the operational benefits of modularity coming from system reconfiguration and therefore they create passive architectures.

\section{Operation scenario, design requirements}

We consider an earthquake event similar to that in Amatrice, Italy, in 2016 where UAVs and UGVs were deployed after the structural analysis tasks (Kruijff-Korbayová et al., 2016). We design a modular autonomous vehicle fleet consisting of UAVs and UGVs to support rescue teams and minimize the work load of rescuers. We define the needs in a particular earthquake scenario and translate them to a set of design fleet objectives and constraints. The scenario assumes a city like Urbino, Italy, with a population of 15000 , with narrow roads and old buildings that have collapsed. The rescue forces do not initially know which buildings are collapsed and where people may be submerged in the rubble. About 50 people are reported missing and the communication network does not work. A large building in the city center is heavily damaged and in danger of collapsing. There are blocked roads, damaged water and gas pipes, and injured people needing medical care. The operational area covers $4 \mathrm{~km}^{2}$.

The tasks to be performed by the rescue fleet are grouped into five categories:

1. Documentation

a. Aerial imaging to locate areas heavily damaged and report to the rescue forces to help with coordination of the rescue mission.

b. 3D screening to determine safety for personnel to enter buildings, before or after rescue.

2. Search missions

a. Aerial search for submerged people using thermal cameras.

b. Ground search for submerged people with radar sensing heartbeats or breathing. 
3. Transportation missions

a. Aerial transportation of light goods including medicine, vaccines, blood, water, etc.

b. Ground transportation of rubble and tools.

4. Communications network

a. Restoration of the broken communication network.

5. Gas sensing

a. Aerial and ground detection of gas leaks with proper sensors.

We extract several design attributes for the fleet. An autonomous fleet must be designed for easy transportation to the disaster site. Therefore, the required space for transportation and the weight of the entire fleet should be minimized. An efficient mission demands long operational times for UAVs and UGVs. Lightweight vehicles decrease energy consumption and increase time of operation. We define a minimal operation time of 30 minutes for all vehicles. For an overall mission period of 48 hours, recharging batteries is necessary. Instead of making the vehicles unavailable during the charging process, we require the battery to be replaceable. We further require the autonomous UGVs to replace the batteries of the UAVs without any human intervention, making the teaming between vehicles an essential design element. UAVs and UGVs must arrive at their destination as quickly as possible, so individual vehicle speed should be maximized. The vehicles must perform under changing weather conditions and terrain, necessitating weather protection, lighting equipment to operate in the rubble, and UGV drivetrain and clearance appropriate for moving through the rubble. Reliability, durability and easy repairs are also needed. Allowing upgrades to the vehicle electronics is desirable.

The tasks identified require specific sensors on the vehicles. Deployment outdoors and indoors requires autonomous navigation indoors when GPS signal is not available. Aerial imaging requires highdefinition (HD) cameras. To get high-quality footage, the vibrations of the frame and the attachment of the camera must be as low as possible. Creating 3D-models of buildings requires appropriate 3D scanners. Search missions need radars, thermal cameras, and gas detection sensors. Transportation missions require sufficiently high payload. The time for securing the payload on the vehicle has to be as low as possible. Wi-Fi devices are needed for communications.

\section{Design process}

A common element in design processes is iteration whether in formal standards (e.g., VDI, 1993; INCOSE, 2015) or in simplified educational models (e.g., Papalambros, 2015). The simplified process consists of five phases, namely, (1) definition where the right problem based on user need/wants and associated attributes are defined, (2) divergence where several alternative solutions are created, (3) transition where detailed selection criteria are refined, (4) convergence where a solution is selected, and (5) prototyping where a prototype of the selected solution is built to verify and validate the solution. The model allows iterations throughout.

The above process does not differentiate between conceptual and embodiment (detailed) design explicitly, therefore we extend the process model as shown in Figure 1. This process model has eight phases: problem definition (Phase 1), conceptual design (Phases 2-4), embodiment (Phases 5-7) and prototyping (Phase 8) where both conceptual and embodiment design have their own divergence, transition and convergence phases. We explicitly show the iterations within and among conceptual and embodiment stages. Note that iterations take place on prototypes of varying fidelity but only a final detailed prototype is shown explicitly to acknowledge the need for verification and validation. We adopt this model here as balancing simplicity and comprehensiveness. We apply this process to develop both modular and integrated design solutions. While the process remains the same for both types of solutions, the difference is observed at the convergence phase where modular designs may perform better or worse compared to integrated solutions. 


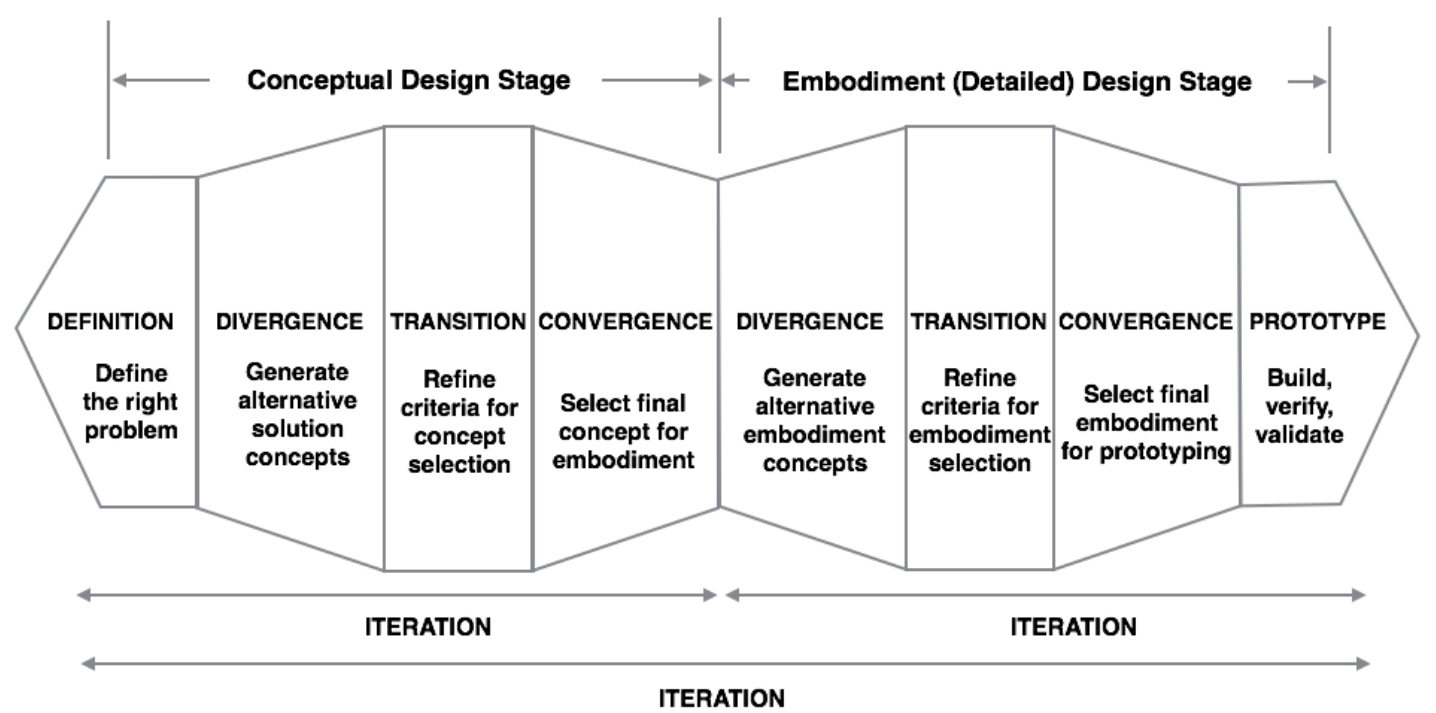

Figure 1. The design process

\section{Conceptual design}

Conceptual design involves functional development of UAV and UGV concepts in view of the desired design attributes. Both ideation techniques and computational methods are employed to create multiple concept alternatives. A concept selection method such as a Pugh Chart is used to select a concept for further development.

\subsection{Concept generation}

Following the above process model, we performed two brainstorming sessions with a group of eight design researchers at the University of Michigan. The first session was structured as an open-ended session with researchers working in pairs and developing mind maps. A second group discussion session was conducted converging to two conceptual design solutions for the overall fleet.

In addition to brainstorming, we used a DSM-based approach to create additional design alternatives. We identified 17 physical elements in the UAVs and UGVs combined, created the DSM based on interactions among the (functional or physical) elements of the fleet design, and defined multiple objectives such as minimizing the size of the modules and minimizing the interactions among modules to draw boundaries in the DSM that define the modules (Eppinger and Browning, 2012). We omit the DSM presentation here for brevity. We then formulated an optimization problem to minimize the two objectives namely, sum of module sizes and the number of required interactions/interfaces among modules, with respect to the clustering the components. Solving the multiobjective optimization problem, we obtained 30 more concepts.

Here we discuss six representative designs. Concept 1 generated in the brainstorming session places a Wi-Fi module on a blimp due to the stationary nature of the mission. This concept assumes separate platforms with essential electronics and drivetrain components for UAVs and UGVs. Since gas sensing is required for all vehicles, the platforms also contain gas sensors. Battery and all other mission-related sensors are defined as separate modules in this concept. Concept 2 is similar to Concept 1 except it combines sensors required by the same mission into a single module. It defines a search module with thermal camera, radars, documentation module with HD camera and another module with 3D scanner, and a transportation module. Concepts 3-6 are created by the DSM method. Concept 3 is a non-modular, multifunctional vehicle that can perform both aerial and ground missions with all the necessary components available on the vehicle. Concept 6 is at the opposite end of the spectrum with almost all elements separately defined as modules including the Wi-Fi device. It only combines motors, ECS and battery as a module. Concept 4 has separate platforms for UAVs and UGVs with essential electronics and 3D scanners, payload attachment and thermal cameras. Drivetrain and other mission sensors are located in separate modules. Concept 5 has motors and rotors for UAVs, and rolling gear for UGVs, the 
Wi-Fi device and HD camera as separate modules, and a platform contains everything else. The assignment of elements to the modules in these concepts is shown in Table 1.

Table 1. DSM concepts configurations

\begin{tabular}{|c|c|c|c|c|c|c|c|c|c|c|c|c|c|c|c|c|c|}
\hline Designs & $\mathbf{E}$ & $\begin{array}{l}\mathbf{E} \\
\mathbf{2}\end{array}$ & $\mathbf{E}$ & $\mathbf{E}$ & & $\mathbf{E}$ & $\begin{array}{l}\text { E } \\
7\end{array}$ & & $\begin{array}{l}\mathbf{E} \\
\mathbf{9}\end{array}$ & $\begin{array}{c}\mathbf{E} \\
\mathbf{1 0}\end{array}$ & $\begin{array}{c}E \\
11\end{array}$ & $\begin{array}{c}E \\
12\end{array}$ & $\begin{array}{c}\mathbf{E} \\
13\end{array}$ & $\begin{array}{c}E \\
14\end{array}$ & $\begin{array}{c}\mathbf{E} \\
15\end{array}$ & $\begin{array}{c}E \\
16\end{array}$ & $\begin{array}{c}\mathbf{E} \\
17\end{array}$ \\
\hline Conc. 4 & 1 & 1 & 1 & 2 & 2 & 1 & 2 & 3 & 4 & 5 & 2 & 2 & 6 & 7 & 8 & 8 & 2 \\
\hline Conc. 5 & 1 & 2 & 3 & 3 & 3 & 3 & 3 & 4 & 3 & 5 & 3 & 3 & 3 & 6 & 2 & 2 & 6 \\
\hline Conc. 6 & 1 & 2 & 2 & 3 & 4 & 2 & 5 & 6 & 7 & 8 & 9 & 10 & 7 & 11 & 12 & 12 & 13 \\
\hline
\end{tabular}

$\begin{array}{lllllll}\text { E1 } & \text { Rotor } & \text { E6 } & \text { Battery } & \text { E11 } & \text { 3D Camera } & \text { E16 Rolling Gear } \\ \text { E2 } & \text { Motor } & \text { E7 } & \text { Flight Contr. } & \text { E12 } & \text { Thermal Camera } & \text { E17 Payload attachm. } \\ \text { E3 } & \text { MCU } & \text { E8 } & \text { GPS } & \text { E13 Radar } & & \\ \text { E4 } & \text { Frame } & \text { E9 } & \text { LQ Camera } & \text { E14 WiFi-Device } & & \\ \text { E5 } & \text { Landing Gear } & \text { E10 } & \text { HQ Camera } & \text { E15 } & \text { Climbing Gear } & \end{array}$

The six concepts represent a range from highly modular to integrated design. We compare these designs using a fleet performance simulation that models the operation of multiple UAVs and UGVs working together in the field. The simulation model identifies the potential costs and benefits of using designs with different levels of modularity.

\subsection{Fleet performance simulation}

We developed a fleet simulation model that evaluates the performance of the functional fleet design concepts under the earthquake scenario in Section 2. We employed a time-marching simulation where locations of missing people, collapsed buildings, and gas leaks are random, and the locations of buildings for 3D scanning are predefined. We evaluated each design concept under the aforementioned scenario by operating the entire vehicle fleet throughout the search, rescue and documentation missions. We performed 100 Monte Carlo simulations using several criteria: (1) total redundancy as a measure of the number of components not required by the mission but available on the vehicle; (2) number of vehicles used throughout the simulation; (3) redundancy per vehicle; (4) number of reconfigurations necessary to assemble and disassemble modules; (5) total simulation time needed to find all 50 missing people; and (6) total number of battery recharges for all vehicles. In the simulation, missions may trigger each other. For instance, identifying a person with a thermal camera requires a detailed search with radar. If radar is not available on the vehicle, another vehicle needs to be called which affects the number of vehicles needed and redundancy metrics.

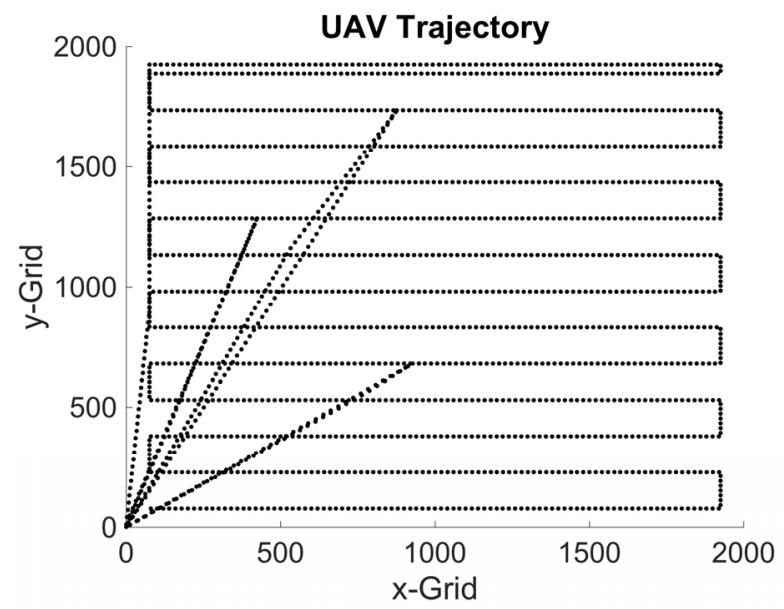

Figure 2. Trajectory a UAV without replaceable battery searching for people 
UAVs and UGVs search for missing people following predefined trajectories. Figure 2 shows how a UAV without battery replacement capability scans the area and comes back for battery recharge. We see from the simulation results that if the automated battery replacement is not an option, UAVs spend a lot of time for returning back to the base station for recharging. Therefore, it is essential to design the UAV and UGV teaming as a physical system and a control system to maximize fleet performance. Vehicles also return to the base station for reconfigurations. Concepts that minimize the number of necessary reconfigurations perform better in the fleet simulation.

\subsection{Concept selection}

Following the simulation results, we defined further criteria to inform the design selection process. We used the chart shown in Table 2 to compare the six concepts evaluated on a scale of 1-5. We divided the performance in the fleet simulation further into sub-criteria based on the level of information provided by the simulation model. Additional criteria shown in Table 2 in bold cannot easily be evaluated by a simulation and hence require engineering judgement at the early design stages due to lack of information related to embodiment. We assign subjective weighting based on the pairwise comparison of each metric on a scale of three levels (more important, equally important, less important) using engineering judgement. Modular design makes a significant difference at this point. Modular systems are easier to maintain and upgrade, and enable lightweight design. Also, the manufacturing process may benefit from choosing modular solutions. However, the development effort for modular design could be higher compared to integrated solutions.

We selected Concept 2 created by brainstorming. Interestingly, the designs created by the DSM method (Concepts 3-6) were judged inferior, likely because modularity decisions based on predefined interactions among components do not yield optimal results when fleet-level operational metrics are considered. The non-modular design in Concept 3 performs second best in the fleet simulation only surpassed by Concept 2 . To fully benefit from modularity, the design concepts must be evaluated at the fleet level considering factors beyond those in the DSM.

Table 2. Comparison of concept alternatives

\begin{tabular}{c|c|cccccc} 
Criteria & Weight & $\mathrm{C} 1$ & $\mathrm{C} 2$ & $\mathrm{C} 3$ & $\mathrm{C} 4$ & $\mathrm{C} 5$ & $\mathrm{C} 6$ \\
\hline Fleet simulation performance & $\mathbf{2 5}$ & 2,3 & 4,2 & 3,9 & 1,5 & 2,7 & 3,5 \\
\hline Total redundancy & 27,5 & 4 & 5 & 3 & 1 & 2 & 3 \\
Number of vehicles & 27,5 & 1 & 4 & 5 & 2 & 2 & 4 \\
Reconfigurations & 20 & 1 & 4 & 5 & 1 & 4 & 4 \\
Simulation time & 12,5 & 2 & 4 & 3 & 2 & 4 & 4 \\
Redundancy/\# of vehicles & 7,5 & 5 & 4 & 2 & 1 & 2 & 2 \\
Number of recharges & 5 & 2 & 3 & 3 & 3 & 2 & 2 \\
\hline Adaptability & $\mathbf{2}$ & 3 & 3 & 4 & 3 & 3 & 3 \\
Maintainability & $\mathbf{2 0}$ & 5 & 4 & 2 & 3 & 3 & 3 \\
Transportability & $\mathbf{2 0}$ & 3 & 5 & 4 & 1 & 2 & 4 \\
Upgradability & $\mathbf{9}$ & 4 & 4 & 2 & 3 & 3 & 3 \\
Development effort & $\mathbf{1 1}$ & 2 & 3 & 4 & 3 & 3 & 3 \\
Manufacturing & $\mathbf{2}$ & 3 & 3 & 2 & 3 & 3 & 3 \\
Vehicle weight & $\mathbf{1 3}$ & 5 & 4 & 1 & 3 & 4 & 3 \\
\hline Sum & 510 & 352 & 419 & 304 & 229 & 290 & 338
\end{tabular}

\section{Embodiment design: UAV}

Next, we developed the embodiment for the modular UAV design (modular UGV is a future task). Following the process model again, we generated multiple alternatives, refined the selection criteria and made a final choice among the alternatives. 


\subsection{Drivetrain design and hardware selection}

The first step in embodiment design of the UAVs is drivetrain design and hardware component selection. These decisions inform the frame's structural design.

Selection of motors and other drivetrain components depends on the configuration of the multirotor UAVs. We considered quadcopters, hexacopters, and octocopters. Increasing the number of rotors, increases the robustness of the system in case one of the motors malfunctions. The hexacopters and octocopters are still controllable and can continue the mission in such cases. Furthermore, higher payloads can be carried by hexacopters and octocopters. However, the UAVs become heavier and larger with increasing number of rotors, which reduces maneuverability. We selected the hexacopter configuration for maneuverability and robustness, leaving heavier transportation tasks to the UGVs. To select the appropriate set of batteries, motors, rotors, and fitting motor controllers for a hexacopter, we used the online component selection software eCalc commonly used by individual drone developers and enthusiasts (Solution for All, 2017). Weight is a key parameter for component selection that largely depends on the selected battery, the heaviest component. Increasing battery capacity increases the weight and may not always lead to longer flight times. An iteration process was used:

1. Select the appropriate motor.

2. Select the appropriate propeller. Quickly changeable or foldable propellers are preferred.

3. Calculate the thrust for the motor propeller combination using eCalc.

4. Choose the appropriate Electronic Speed Controller (ESC). The speed and efficiency of the controller determines how efficient and stable the drone will fly and how smooth it can be controlled. The maximum current draw is used to choose the correct ECS.

5. Determine the minimum frame size, depending on the size of the propellers. They should not touch each other during operation.

6. Pick an appropriate battery based on voltage, maximum current draw and required flight time.

7. Calculate the total weight.

8. Restart the iteration until the performance is satisfactory.

During these iterations, we determined that propellers larger than 12in provide increased efficiency because the current drawn from the battery is reduced. To drive these larger propellers, slower and larger motors are needed which increase the overall weight of the UAV. The weight of a drone has a positive impact on overall payload and controllability under windy weather, while reducing manoeuvrability and transportability of the drone. The main challenge is to find the optimal size of the propellers to provide the required flight time while considering the implications of drone weight. After several iterations, we chose the DJI brand Snail 2305 motor with the 5048S tri-blade propeller. The battery is the Strix brand power Strix lithium-ion battery with $7.4 \mathrm{~V}$ output and $3200 \mathrm{mAh}$ capacity.

Other hardware decisions include selection of flight controller and a microprocessor to enable autonomous operation. To increase commonality among UAVs and UGVs, the sensors and microcontrollers should be compatible for both systems. We chose a combination of Pixhawk 2.1 as the flight controller and Raspberry Pi as the microcomputer for autonomy functions. The benefits of using the Pixhawk are its open source code and robustness in flight operation and flexibility for a variety of sensors. The Raspberry Pi can be easily connected to the Pixhawk and offers a Linux-based open source software with machine learning capabilities. For the required sensors listed in Section 2, we chose the LiDAR Lite because it comes as plug-and-play module that can be easily connected to the Pixhawk and programmed to measure distance from ground and surrounding objects for indoor navigation. For aerial imaging, we chose a dedicated HD camera such as Gopro Hero 5 and a dedicated thermal camera such as Flir Vue Pro. These two cameras can both record GPS data when taking pictures that can be used to identify the precise locations of the points of interest. We choose MQ-2 gas sensor due to its reliability and ease of integration with the Raspberry Pi.

\subsection{Frame design - first generation}

For the frame design we focused on the mechanical interface between the module holder and the UAV platform, since electrical interfaces are standardized and easy to implement. The interface must be 
lightweight, durable, withstand forces during operation and mounting, and allow for module replacement by a single person intuitively and with no additional tools.

Brainstorming resulted in several alternatives: Multiple screw attachment; single threaded platform and module attachment; use of lock ring or clamping set for force-fitting; bayonet catch combining form and force-fitted solutions. Additional alternatives were latch, lashing strap, hose coupling/ quick lock coupling, snap buckle, seatbelt-like buckle, mechanical/electrical/magnetic interface, etc. Several concepts were eliminated as not meeting the desired attributes. The remaining ones were compared based on cost, complexity, replacement time, and durability. A latch concept was finally selected.

Frame design concepts were developed next. Foldable and unibody solutions were considered including having battery and flight controller mounted on a base plate, with six arms that can fold sideways or downwards for storage; or a unibody frame with six non-foldable arms and the module holder attached onto it. Evaluation considering weight, ease of transportation, durability and reliability during operation, fragility of joints, and vibration during flight, all concepts were eliminated but a new one with arms bolted but easily detachable from the main plate was created and selected. In this frame design, the electronics are fitted onto the main plate and the motors are attached to mounts on the end of the arms. In this design, the module interface was placed on top of the main plate and the battery at the bottom, integrated with the landing gear considering all different mission modules. Some tasks, like 3D scanning, demand free sight around and above them. During the 3D scanning task, the drone needs to land and motors are turned off during the scanning. This saves energy and delivers better quality scans due to the absence of vibrations. The battery was placed below the main plate to facilitate autonomous replacement by the UGVs. Removing the classical landing gear with long landing skids made the overall design more compact and the battery more accessible.

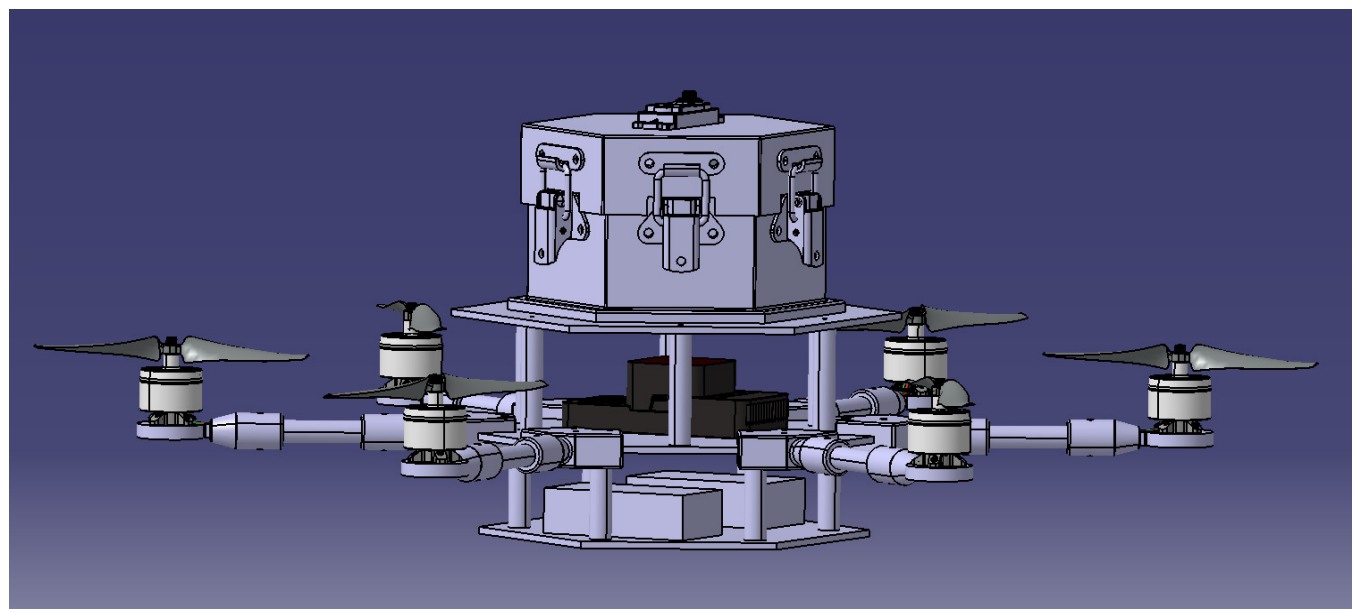

Figure 3. First-generation frame embodiment design

Figure 3 shows the three-layered embodiment design. Plates and module holder are hexagonal; the two batteries are attached; flight controller and electronics are located at the middle layer. The module holder with a representative module attached through six latches is placed on top. Motor and rotor assemblies are attached to the motor mounts that are bolted on a carbon fiber tube. Six plastic columns connect the three layers. This design is lightweight with easily accessible parts. However, the components are not sealed from external influences, and the column connection cannot withstand torsion during assembly or operation; the latch design and hexagonal shape are inefficient. The next design iteration considers these drawbacks.

\subsection{Frame design - second generation}

The second-generation design followed more detailed structural analysis. Figure 4 shows a design with circular shape to increase space efficiency, and an enclosed body to protect the electronics. There are no multiple layers and it is rigid in torsion. A module holder with a triangular shape and rounded edges reduces the number of latches to three. 
We used finite element analysis to determine location and magnitude of maximum stresses under various load cases, including full motor thrust and impact loads on the module holder and batteries due to crash. For example, the crash simulation showed high stress areas in the radius between the mechanical interface and the rest of the body as shown in Figure 4. Based on this analysis, the final design shown in Figure 5 was created with improved connector, module holder, and additional supports at the high stress areas.

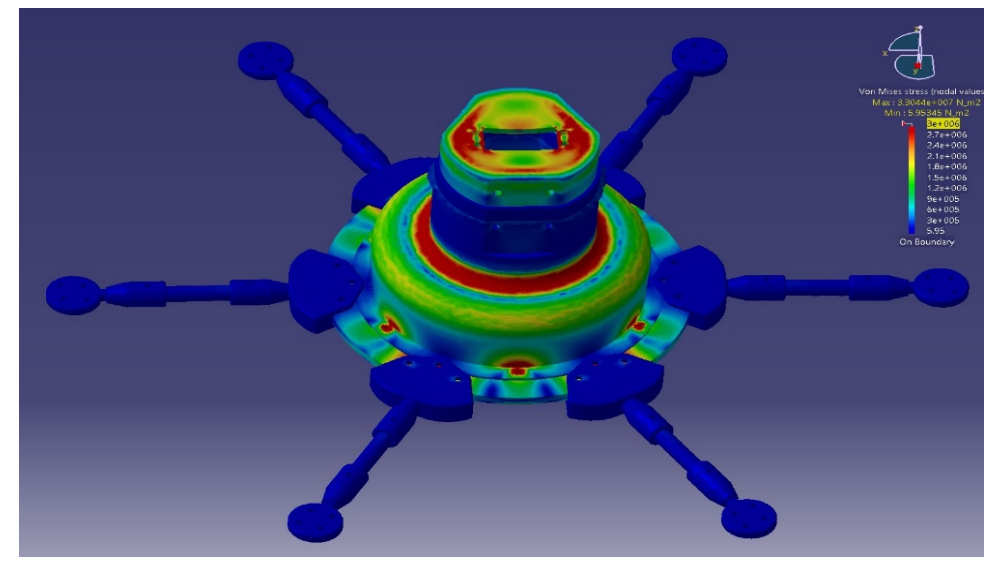

\section{Figure 4. Stress distributions on the initial second-generation embodiment design}

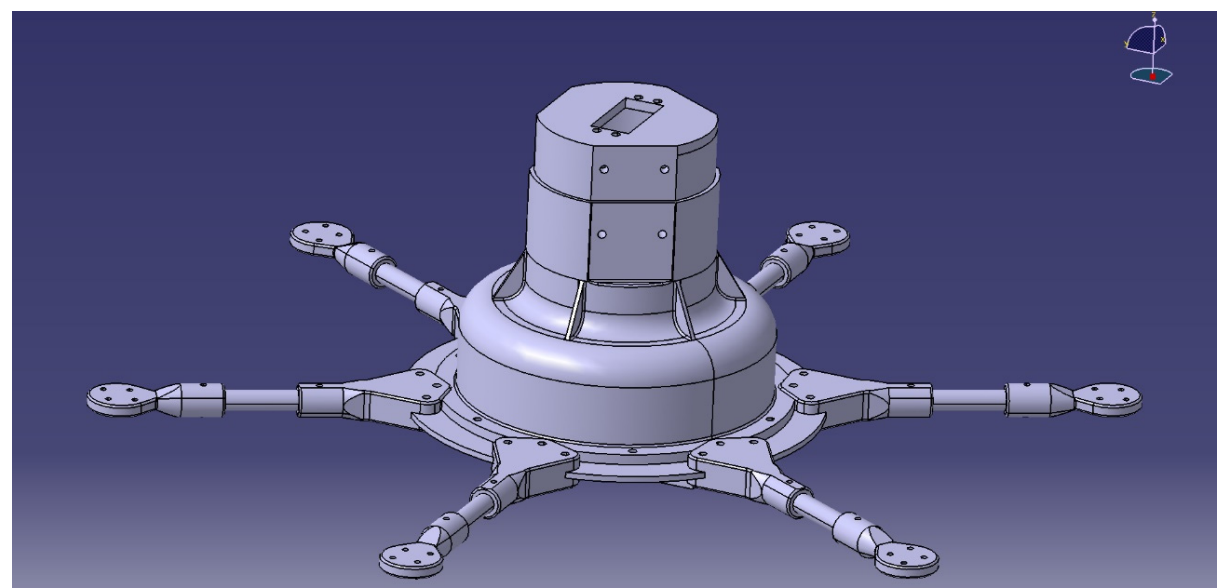

Figure 5. Second generation frame embodiment design

The design features landing directly on the batteries to enable automated battery replacement by the UGV. A functional design for the battery casing has been developed using a rail system to allow easy replacement; its detailed design must be made in conjunction with the UGV embodiment and is not included here.

\section{Prototype: UAV}

We built multiple prototypes during the design process with increasing degree of fidelity to guide both conceptual and embodiment design decisions, see Figure 6 for an example of an early one. We started with a conventional hexacopter to understand key issues such as packaging and power consumption, then we developed the modularity functionality into that prototype in conjunction with the design decisions described in earlier sections. We reused components between different prototypes to the extent possible. We facilitated this process by modular connectivity within the electronic circuitry including the connections for the ESCs, the motors, and the power distribution board. This plug-and-play capability of the electronic components significantly decreases fabrication, assembly, and repair effort during tests. 


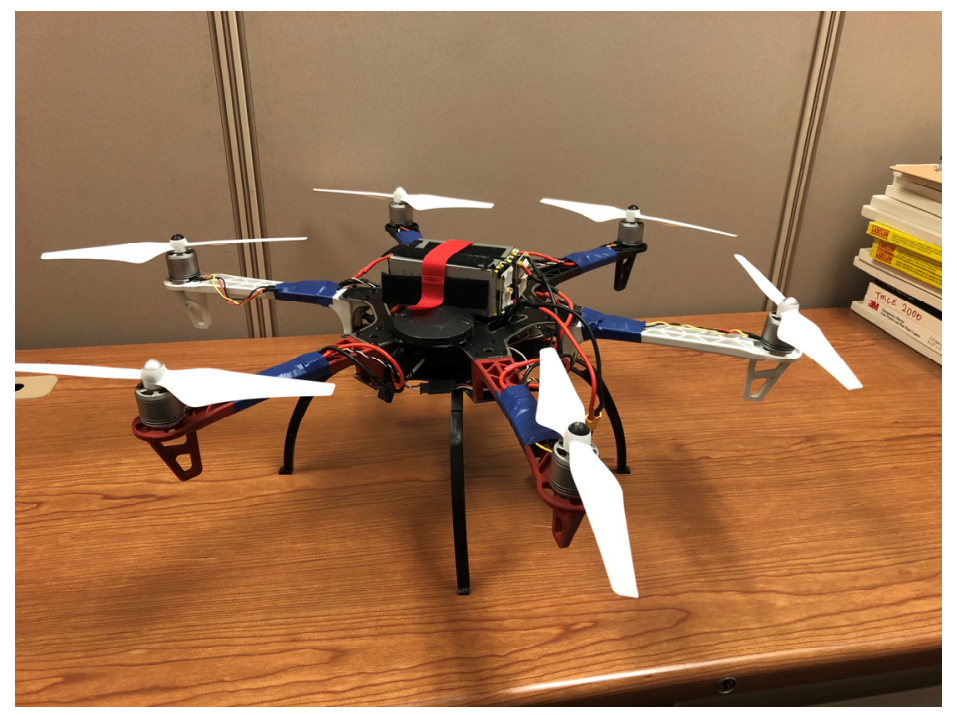

Figure 6. Example of an early prototype built for design exploration

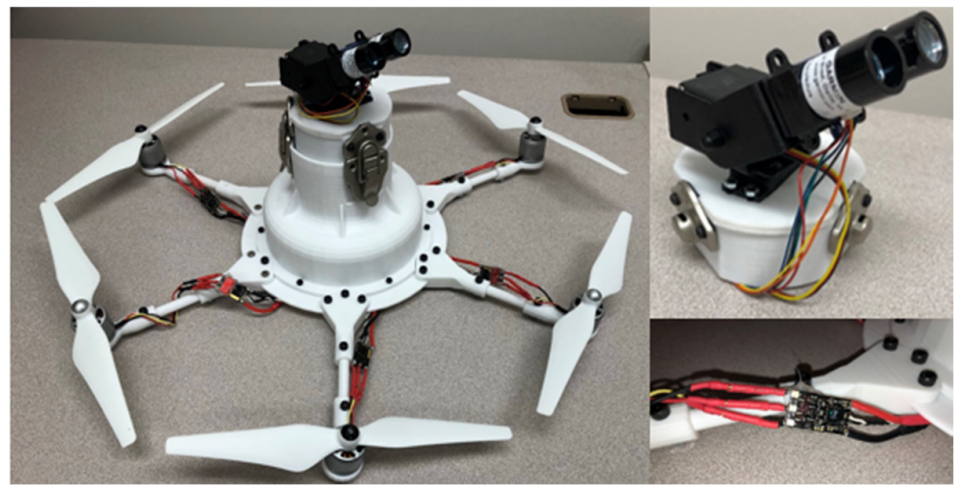

Figure 7. Prototype of the second-generation design embodiment

We built the final prototype with 3D printing for the UAV frame using polycarbonate. We printed the main plate, main body, module holder, LiDAR module, as well as motor mounts, arm tubes, and arm connectors. This prototype, shown in Figure 7, demonstrates the feasibility of the modular design, mechanical interfaces and packaging. We prototyped the LiDAR scanning module by connecting the LiDAR Lite to servos and a microcontroller, which allows scanning the surroundings and creating a 3D plot of point-cloud data.

\section{Future work: UGV design}

A major benefit of the proposed fleet configuration is the teaming between UAVs and UGVs. We can combine their different capabilities to maximize fleet performance and efficiency. The next step for fleet design development is the detailed design of the UGV embodiment. Two main issues must be addressed: the off-road capabilities of the UGVs and the interface with the UAVs.

For the off-road capabilities, body stiffness, ability to move through rubble and obstacles in different shapes, and mobility under different terrains must be designed to achieve the required performance.

Another important element in teaming is the replacement of the UAV batteries by the UGVs. For that purpose, the battery casing for the UAV and the replacement mechanism in the UGV must be designed together to make the replacement as easy and quick as possible. The initial functional design we develop at the conceptual design stage features the battery to slide through rails mounted in the casing. In that way, the UGV can quickly remove the old battery and push the new one in. This initial idea might be iterated based on the replacement mechanism solutions developed for the UGV. For instance, we assume 
in this initial design that the UAV can land on the UGV and lock in a predefined position. If the UGV embodiment design cannot assure a precise landing, alternative solutions might be necessary.

A further challenge is to ensure communication between the UAVs and the UGVs, so that they can exchange information for a proper cooperation throughout the mission for task sharing as well as battery replacement. The UGV embodiment design should also be compatible with the modules designed for the UAVs to enable module sharing. It might be possible that the UAV design can also be iterated based on the decisions made for the UGV design. The UGV embodiment should be designed following the same process given in Figure 1.

\section{Conclusions}

In this paper, we demonstrated a combined functional and physical design process for a fleet of modular autonomous UAVs and UGVs for disaster relief scenarios. We developed an earthquake scenario to extract design requirements. We followed an iterative process to develop design concepts for the UAVs and UGVs together and designed a detailed embodiment for the UAVs. We evaluated the performance of modular and non-modular concepts in a fleet simulation. We demonstrated the feasibility of the final UAV design with a prototype and highlighted important challenges to be addressed by the UGV design in a future study.

Results showed that making modularity decisions based on predefined interactions using a DSM optimization may not always lead to optimal designs in terms of operational performance, in part due to inability to represent all design attributes in the DSM. Modularity decisions must be made considering the operation of the entire fleet as a function of time. We also showed the synergy between modularity and autonomy at a fleet-level in the automated replacement of UAV batteries by UGVs that led to improved fleet performance. Such improvement can only be achieved by having both modularity and autonomy built into the overall system design. Future design iterations may consider automated reconfiguration of modular UAVs that can further reduce dependency on human operators.

\section{Acknowledgment}

This work was partially supported by the Donald C. Graham Chair Endowment at the University of Michigan and the Automotive Research Center, a US Army Center of Excellence in Modeling and Simulation of Ground Vehicle Systems headquartered at the University of Michigan. This support is gratefully acknowledged. We also wish to thank colleagues at the Singapore University of Technology and Design, and Luciënne Blessing in particular for discussions resulting in the simplified design process model we used in this study.

\section{References}

Bayrak, A.E., Collopy, A.X., Papalambros, P.Y. and Epureanu, B.I. (2017), "Multiobjective optimization of modular design concepts for a collection of interacting systems", Structural and Multidisciplinary Optimization, Vol. 57 No. 1, pp. 1-12. https://doi.org/10.1007/s00158-017-1872-4

Borjesson, F. and Hölttä-Otto, K. (2014), "A module generation algorithm for product architecture based on component interactions and strategic drivers", Research in Engineering Design, Vol. 25 No. 1, pp.31-51.

Câmara, D. (2014), "Cavalry to the rescue: Drones fleet to help rescuers operations over disasters scenarios", IEEE Conference on Antenna Measurements \& Applications (CAMA), Antibes Juan-les-Pins, France, November 1619, 2014, IEEE, pp. 1-4. https://doi.org/10.1109/CAMA.2014.7003421

Eppinger, S.D. and Browning, T.R. (2012), Design structure matrix methods and applications, MIT press, Cambridge, MA.

Fujita, K. and Yoshida, H. (2004), "Product variety optimization simultaneously designing module combination and module attributes", Concurrent Engineering, Vol. 12 No. 2, pp. 105-118. https://doi.org/10.1177/1063293x04044758

Gershenson, J.K., Prasad, G.J. and Zhang, Y. (2003), "Product modularity: definitions and benefits", Journal of Engineering design, Vol. 14 No. 3, pp. 295-313. https://doi.org/10.1080/0954482031000091068

Huang, C.C. and Kusiak, A. (1998), "Modularity in design of products and systems", IEEE Transactions on Systems, Man, and Cybernetics: Systems and Humans, Vol. 28 No. 1, pp. 66-77. https://doi.org/10.1109/3468.650323

INCOSE (2015), Systems Engineering Handbook: A Guide for System Life Cycle Processes and Activities, 4th ed., INCOSE, Wiley, New Jersey. 
Iqbal, U., Shah, S.I.A., Jamil, M., Gillani, S.O. and Ayaz, Y. (2015), "Selection of unmanned aerial system (uas) for disaster relief operations: A comparison”, Journal of Science International, Vol. 27 No. 4, pp. 3199-3203.

Koh, E.C.Y., Caldwell, N.H.M. and Clarkson, P.J. (2013), "A technique to assess the changeability of complex engineering systems”, Journal of Engineering Design, Vol. 24 No. 7, pp. 477-498. https://doi.org/10.1080/09544828.2013.769207

Koh, E.C.Y., Förg, A., Kreimeyer, M. and Lienkamp, M. (2015), “Using engineering change forecast to prioritise component modularisation”, Research in Engineering Design, Vol. 26 No. 4, pp 337-353.

Kruijff-Korbayová, I., Freda, L., Gianni, M., Ntouskos, V., Hlaváč V. et al. (2016), "Deployment of ground and aerial robots in earthquake-struck Amatrice in Italy". IEEE International Symposium on Safety, Security, and Rescue Robotics, Lausanne, Switzerland, pp. 278-279. https://doi.org/10.1109/SSRR.2016.7784314

López, L.B., van Manen, N., van der Zee, E. and Bos, S. (2017), "DroneAlert: Autonomous Drones for Emergency Response”, In: Nurmi, J., Lohan, E.-S., Wymeersch, H., Seco-Granados, G. and Nykänen, O. (Eds.), MultiTechnology Positioning, Springer, pp. 303-321. https://doi.org/10.1007/978-3-319-50427-8_15

Measure (2015), Drones for Disaster Response and Relief Operations, Measure, Washington DC.

Papalambros, P.Y. (2015), "Design Science in Design Education”, Proceedings of the XI Mudd Design Workshop on Design Thinking in Design Education, Harvey Mudd College, Clairmont, CA, May 28-30, 2015, pp. 28-30.

Simpson, T.W., Jiao, J., Siddique, Z. and Hölttä-Otto, K. (2014), Advances in product family and product platform design, Springer, New York.

Solution for All (2017), eCalc. [online] Markus Müller. Available at: https://www.ecalc.ch

Ulrich, K. (1994), "Fundamentals of Product Modularity”, In: Dasu S., Eastman C. (Eds.), Management of Design, Springer, Dordrecht. https://doi.org/10.1007/978-94-011-1390-8 12

VDI (1993), Richtlinie 2221 - Methodik zum Entwickeln und Konstruieren technischer Systeme und Produkte, VDI- Verlag, Düsseldorf.

Andreas Christian Gärtner, M. Sc.

University of Michigan, Mechanical Engineering

2350 Hayward Street, 48109-2125 Ann Arbor, United States

Email: ChristianGaertner@gmx.de 